\title{
Documentos
}

\section{RELACIÓN ENTRE RENDIMIENTO LABORAL Y SÍNDROME PREMENSTRUAL}

\author{
Natalia Espina V.a, Alejandra Fuenzalida A. ${ }^{b}$, María Teresa Urrutia S. ${ }^{b}$ \\ aEnfermera Matrona. benfermera Matrona, Departamento de Salud de la Mujer, Escuela de Enfermería, Pontificia \\ Universidad Católica de Chile.
}

\begin{abstract}
RESUMEN
El Síndrome Premenstrual (SPM) afecta a gran cantidad de mujeres en edad reproductiva y se caracteriza por una repetición cíclica de síntomas físicos y psicológicos que, en algunos casos, pueden llegar a ser lo suficientemente severos como para interferir en los patrones de vida. El objetivo de esta revisión bibliográfica es conocer cómo afecta el SPM el rendimiento laboral de las mujeres. A pesar de las significativas repercusiones personales y económicas, existen pocas investigaciones que estudien la relación entre SPM y rendimiento laboral. Sin embargo, la literatura es concluyente en señalar que la productividad laboral es un importante dominio de la vida de la mujer adversamente afectado por el SPM, manifestado en un aumento de la tasa de ausentismo laboral y reducción de la productividad. Se concluye, según la literatura revisada, que el SPM afecta el rendimiento laboral, lo que sugiere a los profesionales de la salud tomar un rol activo en la derivación y tratamiento oportuno, para de esta forma lograr un aumento en la productividad laboral de las mujeres.
\end{abstract}

\section{PALABRAS CLAVE: Síndrome premenstrual, productividad laboral}

\section{SUMMARY}

The premenstrual syndrome (PMS) affects a large quantity of women of reproductive age, and it characterizes because of a cyclic recurrence of physical and psychological simptoms that, in some cases, might become severe enough as to interfere with a persons life pattern. The objective of this paper is to gather a bibliographic revision that will lead us to know how PMS affects the working performance of women. Although the repercussions in this matter are very significant, there are only a few studies about the relation and interdependence between this syndrome and the efficiency at work. However, some literature establishes that the PMS largely affects the working productivity of women, which is manifested in reduction of productivity and larger rates of working absenteeism. The conclusion we support after having checked papers, reports and literature written about PMS is that this syndrome does affect the working performance of those how suffer it, by enhancing the rates of labor absenteeism and reducing productivity at work. This suggests that health professionals should take a more active role in the treatment of this disease, in order to achieve the goal of increasing the productivity at work for women.

KEY WORDS: Premenstrual syndrome, work productivity 


\section{INTRODUCCIÓN}

El deterioro de la salud conduce a menudo al debilitamiento del trabajo, ya sea en forma de ausentismo o de reducción de la productividad. La productividad relativa a la salud ha sido reconocida cada vez más como un componente esencial de la carga asociada a una enfermedad (1). Un problema común de las mujeres en edad reproductiva corresponde a los síntomas físicos y emocionales que ocurren durante el ciclo menstrual (2), los que a menudo son clasificados bajo el término genérico "Síndrome Premenstrual" (SPM) (3). Diversos autores concuerdan que estos síntomas pueden llegar a ser lo suficientemente severos como para interferir en diversas áreas de la vida de la mujer, entre ellas las relaciones interpersonales, actividades sociales y desempeño laboral (4-9).

Las investigaciones en relación al SPM han ido en aumento, éstas más bien estudian la etiología, fisiopatología y las diferentes alternativas de tratamiento, siendo escasas las publicaciones que estudian la relación entre SPM y ámbito laboral. Esta revisión bibliográfica pretende dar a conocer de qué manera afecta el SPM en el rendimiento laboral, con el propósito de enriquecer el conocimiento en torno al tema, brindando una perspectiva hasta el momento poco valorada y/o discutida de éste.

\section{ANÁLISIS DEL TEMA}

El tempestuoso comportamiento premenstrual fue descrito por primera vez por Hipócrates, quien atribuyó los síntomas al "escape agitado que buscaba la sangre de la matriz". Sin embargo, fue en los años 30 cuando el síndrome de tensión premenstrual fue delineado, coincidiendo posiblemente con la entrada de las mujeres a la fuerza laboral (10). La American College of Obstetricians and Gynecologists (ACOG) es optimista en señalar que sólo 5 a $10 \%$ experimentan síntomas bastante severos para ser debilitantes (11), recientes publicaciones revelan que entre el 23 y $67 \%$ de las mujeres son portadoras de sintomatología premenstrual moderada a severa (5-7) las que resultan en disminución del funcionamiento y deterioro de las relaciones interpersonales $(4,7,8)$.

Al estudiar el impacto de la salud en el rendimiento laboral, las investigaciones apuntan a diversas patologías, tanto físicas como psicológicas. Al comparar el SPM con otras patologías o síndromes, el impacto y carga de éste es similar en magnitud a otros desórdenes. Halbreich y cols (12) observaron que el impacto social de las mujeres con síndrome disfórico premenstrual (SDPM) durante la fase lútea fue muy similar al de las mujeres con distimia y depresión mayor crónica.

A pesar de la prevalencia del SPM, aun no ha logrado posicionarse con el peso comparativo de otras enfermedades. De hecho, en un informe de la Organización Mundial de la Salud del año 2001, el SPM no fue incluido dentro de un amplio reporte de desordenes en el área de la salud mental (13). Lolas, ya en el año 1993 (14) nos señalaba que en nuestro país el tema tenía poca difusión científica y que no existía conciencia de la real magnitud de esta patología.

El rendimiento laboral es un importante dominio de la vida de la mujer adversamente afectado por el SPM. Como resultado de la severidad de los síntomas premenstruales surge el ausentismo laboral, dando lugar a repercusiones personales y económicas significativas (15). Una de las principales investigaciones llevadas a cabo en torno al tema corresponde a la realizada por Borenstein y cols (9), quienes exploraron la carga de enfermedad asociada al SPM, valorando la calidad de vida vinculada con la salud, la utilización de recursos sanitarios y el impacto ocupacional, utilizando una combinación del "calendario diario de los síntomas" y los criterios de SPM durante dos ciclos menstruales consecutivos. Estos autores señalan que el SPM se asocia al ausentismo y la reducción de la productividad laboral, ya que las mujeres presentan 5 veces más probabilidades que el grupo control de reportar reducción de la productividad y la mayoría de ellas notaba perder días de trabajo por razones de salud.

En relación al impacto en el funcionamiento laboral, Hylan y cols. (7), señalan que más del $50 \%$ de las mujeres que trabajan fuera del hogar presentan síntomas premenstruales que interfirieren "algo" o "enormemente" con su capacidad de trabajar.

En relación al número de días ausentes producto del SPM, Dean y Borenstein (4), reportaron que del grupo de mujeres laboralmente activas, aquellas que cumplían criterios de SPM en uno o ambos ciclos perdieron un mayor número de días de trabajo que aquellas sin SPM. A pesar que el número de días ausentes al mes entre las mujeres con SPM durante sólo 1 ciclo menstrual fue significativamente mayor que el observado entre mujeres sin SPM (2,5 versus 1,3 días respectivamente), no hubo diferencia significativa entre aquellas con SPM y las sin este síndrome al ser comparadas 
durante dos ciclos. Los autores (4) señalan que la probabilidad de experimentar "alto ausentismo", definido como dos días al menos de ausencia en un mes, fue dos veces mayor en mujeres que presentaban SPM en un ciclo y casi tres veces mayor en mujeres que presentaban SPM en ambos ciclos menstruales. Por otro lado, Hylan y cols (7), señalan que del total de mujeres que reportó interferencia laboral, 11 a $38 \%$ había perdido por lo menos un día de trabajo, y de estas dos tercios habrían faltado entre 1 y 7 días durante el último año. Asimismo, Robinson y Swindler (6) refieren en su artículo un porcentaje similar de ausentismo, detallando que del total de mujeres afectadas, un $14 \%$ se ausentó de 1 a 5 días, 2,3\% de 6 a 10 días, y $0,5 \%$ lo hizo durante 11 a 36 días. Estos porcentajes se vieron significativamente aumentados cuando se trató de SDPM. En nuestro país no se encontraron investigaciones en torno a este tema, salvo una tesis de pre-grado (16), que señala que $22,6 \%$ de las mujeres faltó a su trabajo al menos un día y $54,7 \%$ presentó algún grado de incapacidad a causa de la sintomatología premenstrual.

Con respecto a la relación entre severidad de los síntomas del SPM y ausentismo laboral, se encontró que aquellas mujeres con síndrome severo presentan mayor porcentaje de ausentismo laboral que el grupo con sintomatología menor (5, 6). Sin embargo, el ausentismo es sólo un aspecto de los costos indirectos de la salud. Para obtener una visión más amplia de cómo se ve afectado el rendimiento laboral en relación a los problemas de salud de los trabajadores, otros factores deben ser considerados, entre ellos la productividad, aspecto que fuertemente disminuye en las mujeres que presentan SPM. Este concepto, conocido también como "presenteeism", hace mención a la disminución en la calidad del trabajo en aquellos empleados con problemas de salud que no son lo suficientemente severos como para causar ausencia del lugar de trabajo, el que provoca un gran impacto económico individual y social $(17,18)$. Una de las principales investigaciones que aborda este aspecto corresponde a la realizada por Chawla y cols (5), quienes señalan el impacto de los síntomas premenstruales en el debilitamiento de la productividad y limitación del rol en la fase lútea de la mujer. Los síntomas premenstruales severos y el SDPM fueron significativamente asociados a una reducción del número de horas trabajadas comparado con aquellas mujeres con síntomas mínimos. Durante la fase lútea, las mujeres reportaron ser $19 \%$ menos productivas en su tra- bajo que aquellas con sintomatología mínima. Dentro de cada fase del ciclo menstrual, los puntajes de las mujeres con síntomas mínimos reflejaron una pérdida de productividad lejos más baja que las mujeres con síntomas severos.

Dean y Borenstein (4) señalan que las mujeres con SPM durante un ciclo tienen más probabilidades de disminuir su productividad que aquellas sin SPM ( 7,2 días versus 4,2 días, respectivamente), entendiendo como disminución de la productividad al autoreporte de al menos una reducción del $50 \%$. Del mismo modo, se observó una probabilidad 4,3 y 6 veces mayor de presentar "alta pérdida de productividad" (definida como mayor a 5 días en el mes con una reducción de por lo menos un $50 \%$ ) en las mujeres con SPM en uno o dos ciclos respectivamente, en comparación con aquellas sin SPM.

Borenstein y cols (9) han señalado que el SPM se asocia también a una significativa reducción de la calidad de vida en los aspectos físicos y psicológicos de estas mujeres, al observar puntajes más bajos en el SF-36. Estas diferencias fueron aún más pronunciadas entre las mujeres que presentaban SPM en dos ciclos menstruales consecutivos.

Otro estudio alude además a un debilitamiento generalizado en las actividades en el colegio, hobbies, relaciones interpersonales, actividades sociales y del hogar producto del SPM. El promedio de días por mes con debilitamiento general en las mujeres con SPM durante ambos ciclos fue significativamente mayor que las mujeres con SPM durante sólo 1 ciclo y mujeres sin SPM (22 días, 16,5 días y 9,6 días, respectivamente). Así también, aquellas mujeres con SPM tienen 14 veces más probabilidad de experimentar "alto grado de debilitamiento", definido como un debilitamiento por más de 14 días al mes, que aquellas sin SPM (4). Esto concuerda con lo señalado por Hylan y cols (7), quienes afirman que los rangos más altos en la escala de interferencia de los síntomas premenstruales se obtuvieron en las preguntas acerca de interferencia en el hogar, vida social y escuela.

Específicamente, respecto del rendimiento físico e intelectual de un grupo de alumnas universitarias de nuestro país, se encontró una tesis de pre-grado (19) que señala que $59 \%$ de la muestra percibió una disminución de la concentración, $62 \%$ una disminución de la motivación al estudiar y $54 \%$ de la asistencia a clases. Un estudio realizado en Brasil (20) que intentó comparar el desempeño escolar entre alumnas con y sin SPM, de- 
mostró que el promedio de notas del grupo de alumnas con SPM fue inferior al del grupo sin SPM (4,7 y 5,2 respectivamente); no obstante, este resultado no alcanzó significancia estadística. Del mismo modo, tampoco lo fue al comparar el promedio de notas de las alumnas con SPM dentro y fuera del período premenstrual.

A todo lo anterior, se debería agregar también el incremento de los "costos directos" en salud, definidos como los gastos derivados del diagnóstico y tratamiento de una enfermedad o el pago a proveedores de servicios médicos (15). Las mujeres con SPM utilizan más recursos en salud, lo que se refleja en un aumento de la frecuencia de consultas médicas ambulatorias (9). Lo expuesto anteriormente, permite demostrar el significativo impacto derivado de este síndrome. Sin embargo, cabe destacar que la carga económica dada por los síntomas premenstruales se manifiesta más en términos de disminución del rendimiento laboral que en costos directos (5). Al respecto, Borenstein y cols (15) señalaron que el diagnóstico de SPM fue asociado con un incremento promedio anual de U\$ 59 en relación a costos directos versus un incremento de U\$ 4.333 en relación a costos indirectos por paciente en un año.

\section{DISCUSIÓN}

Los resultados de la productividad son útiles ya que caracterizan el impacto de una determinada enfermedad en el lugar de trabajo y demuestran el efecto del tratamiento frente al rendimiento laboral. La productividad relativa a la salud se ha reconocido cada vez más como componente importante de la carga asociada a una enfermedad dada; sin él, uno no puede determinar confiablemente esta carga (1).

$\mathrm{Si}$ bien es cierto los estudios en relación al SPM han ido en aumento, existen pocas investigaciones con respecto al impacto de la enfermedad en la calidad de vida de las mujeres. Las publicaciones concuerdan en que los síntomas pueden llegar a ser lo suficientemente severos como para interferir en una o más áreas de la vida de la mujer, entre ellas las relaciones interpersonales, sociales y el desempeño laboral (4-9). En países desarrollados, las mujeres abarcan aproximadamente el $70 \%$ de la fuerza de trabajo actual; en Chile las estadísticas de la presencia femenina a nivel de servicio corresponde a un 34\% (21).

Los estudios son concluyentes en afirmar que el SPM tiene un significativo impacto en el rendimiento laboral de las mujeres. De hecho, se su- giere que la severidad de los síntomas premenstruales no sólo resulta en una significativa carga personal y económica a causa de la ausencia laboral, sino que también en una reducción del funcionamiento ocupacional, específicamente en la productividad, provocando un gran deterioro a nivel individual y social (15). La mayoría de ellos señala que la sintomatología premenstrual interfiere en alguna medida con su capacidad de trabajo, siendo directamente proporcional al número de ciclos menstruales comprometidos y al grado de severidad con que se presentan los síntomas. Esto se vio manifestado en el aumento de la tasa de ausentismo (generalmente menor a 7 días) y reducción de la productividad, algunos señalando incluso un porcentaje superior al $50 \%(4-7,9)$. Una interesante reflexión surge al observar que el impacto del SPM provoca una disrupción tal sobre sus vidas, que se extiende más allá de su período sintomático, encontrándose que un $50 \%$ de las mujeres experimentan algún efecto de esta enfermedad por más de dos semanas (9). A pesar de esto, el SPM continúa enfocándose principalmente desde una perspectiva sólo biomédica en desmedro muchas veces de un enfoque holístico, que permitiría abarcar todas las áreas afectadas en la mujer.

Al referirnos al impacto del SPM sobre el rendimiento laboral, es necesario abarcar tanto el ausentismo como la disminución de la productividad. Aunque muchas veces resulta más fácil considerar sólo el primer aspecto, debido a su simpleza al momento de ser cuantificado, no debemos dejar de considerar a aquellas trabajadoras con problemas de salud que no son lo suficientemente severos como para causar ausencia en el trabajo (15). Para esto existen diversos instrumentos, que aunque podrían extrapolarse a cualquier enfermedad, no son comúnmente incluidos en las investigaciones. Sólo un artículo se basó en medidas objetivas para cuantificar la carga de esta enfermedad, al utilizar el EWPS como instrumento de medición (5). Frente a esto, sugerimos la aplicación de indicadores de impacto al momento de evaluar a las mujeres que consultan por SPM, con el objetivo de propender a una valoración mucho más integral en ellas.

Es preciso reflexionar acerca de las metodologías utilizadas para llevar a cabo las distintas investigaciones. Primero, señalar que la mayoría de ellas se basa en análisis retrospectivos, lo que lleva a una menor sensibilidad a los cambios en la presencia y severidad de los síntomas en el ciclo menstrual, subestimando la prevalencia y efectos 
de éste. Por lo anterior, la utilización en una de las investigaciones analizadas del "calendario diario de los síntomas" presenta varias ventajas sobre un análisis retrospectivo, ya que permite evaluar la severidad, el grado de fluctuación de los síntomas y su relación con la productividad sobre un registro diario. Además de ilustrar la relación entre la ocurrencia del síntoma y los cambios de productividad, permite también la comparación de tendencias entre uno y más ciclos menstruales (4). Con lo anterior se disminuye el sesgo dado por la dependencia de períodos largos de memoria y aumentar la eficacia del diagnóstico de SPM, ya que se ha visto que las mujeres tienden a exacerbar negativamente al momento de recordar los síntomas premenstruales en comparación al registro diario (22).

En cuanto a las investigaciones realizadas en nuestro país, no se encontraron recientes publicaciones con respecto al impacto del SPM en el rendimiento laboral de las mujeres que lo padecen, ni más ampliamente en la calidad de vida de éstas. A partir de esto, se podría pensar que el SPM es percibido como una entidad clínica que interfiere poco o nada en los patrones de la vida diaria. Esto es digno de reflexionar, ya que los hallazgos de esta revisión bibliográfica contrastan fuertemente con la mirada un tanto despectiva de la opinión pública en torno al tema, claramente ejemplificada por los medios de comunicación, quienes señalan los síntomas del SPM como triviales y no merecedores de mayor atención ni mucho menos de tratamiento médico. Por lo anterior, es labor fundamental de los profesionales validar la consulta de las mujeres, para que reciban tratamiento precozmente.

Con respecto al manejo de las usuarias que presentan esta patología, el rol profesional en la educación de estas mujeres resulta también esencial, ya que la terapia no farmacológica constituye la primera modalidad de tratamiento frente a pacientes con cuadros menos severos de SPM. La ACOG recomienda cambios en el estilo de vida tales como ejercicio aeróbico, una dieta rica en carbohidratos $\mathrm{y} / \mathrm{o}$ suplementos alimenticios para ayudar a resolver los síntomas premenstruales (11). De esta forma se contribuye al fortalecimiento de las habilidades de autocuidado de las mujeres, otorgando información acerca de cambios en el estilo de vida de acuerdo a las recomendaciones internacionales. Al tratar este síndrome con medidas generales, muchas de ellas podrán experimentar una mejor calidad de vida, lo que se verá reflejado en una mayor productividad en el lugar de trabajo y, a largo plazo, en indicadores a nivel nacional. Por esto, creemos que al reconocer que existe este problema y el efecto ya descrito en la mujer, los profesionales de la salud podemos proporcionar una enorme ayuda a las mujeres afectadas optimizando su capacidad de trabajo.

Dado que son cada vez más las mujeres que trabajan fuera del hogar, se hace imprescindible, en primer lugar, realizar investigaciones a nivel nacional que relacionen el SPM con el rendimiento laboral y, también darle a este síndrome el valor que realmente merece, ya que al parecer, tal como fue señalado por Lolas en 1993 (14), constituye aún un "ignorado problema de salud pública".

\section{BIBLIOGRAFÍA}

1. Prasad M, Wahlqvist $P$, Shikiar R, Tina Shih Y. A Review of Self-Report Instruments Measuring Health-Related Work Productivity: A Patient-Reported Outcomes Perspective. Pharmacoeconomics 2004; 22(4): 225-244.

2. Tempel R. PMS in the Workplace: an occupational health nurse's guide to premenstrual syndrome. AAOHN Journal 2001; 49(2): 72-78.

3. Steiner M. Premenstrual syndrome and premenstrual dysphoric disorder: guiderlines for management. J Psichiatry Neurosci 2000; 25(5): 459-68.

4. Dean B, Borenstein J. A Prospective Assessment Investigating the Relationship Between Work Productivity and Impairment With Premenstrual Syndrome. J Occup Environ Med 2004; 46(7): 649-56.

5. Chawla A, Swindle R, Long S, Kennedy S, Sternfeld B. Premenstrual Dysphoric Disorder: Is There an Economic Burden of Illness? Med Care 2002; 40(11): 1101-12.

6. Robinson R, Swindle R. Premenstrual Symptom Severity: Impact on Social Functioting and Treatment-Seeking Behaviors. J Women's Health Gender Based Med 2000; 9(7): 757-68.

7. Hylan T, Sundell K, Judge R. The Impact of Premenstrual Symptomatology on Functioning and Treatment-Seeking Behavior: Experience from the United States, United Kingdom, and France. J Women's Health Gend Based Med 1999; 8(8):104352.

8. Freeman E, Sammel M, Rinaudo P, Sheng, L. Premenstrual Syndrome as a Predictor of Menopausal Symptoms. Obstet Ginecol 2004; 103(5): 960-66.

9. Borenstein J, Dean B, Endicott J, Wong J, Brown C, Dickerson V, Yonkers K. Health and Economic Impact of the Premenstrual Syndrome. J Reprod Med 2003; 48(7): 515-24.

10. Rapkin A. A review of treatment of premenstrual syndrome and dysphoric disorder. Psychoneuroendocrinology 2003; 28(Supp 3): 39-53.

11. American College of Obstetricians and Gynecologists. Clinical management guidelines for obstetri- 
cians-gynecologists: premenstrual syndome. ACOG 2000; Practice Bulletin 15.

12. Halbreich U, Borenstein J, Pearlstein T, Kahn L. The prevalence, impairment, impact and burden of premenstrual dysphoric disorder (PMS/PMDD). Psychoneuroendocrinology 2003; 28 (Supp 3): 1-23.

13. Organización Mundial de la Salud. Informe sobre la salud en el mundo 2001 - Salud mental: nuevos conocimientos, nuevas esperanzas. 2001, Geneva. Hallado en: www.who.int/whr/2001/es. Acceso 10 marzo de 2005.

14. Lolas J. Síndrome Premenstrual: un Ignorado Problema de Salud Pública (versión electrónica). Rev Méd Chile 1993; 121(5): 560-66.

15. Borenstein J, Chiou Ch, Dean B, Wong J, Wade S. Estimating Direct and Indirect Costs of Premenstrual Syndrome. J Occup Environ Med 2005; 47(1): 2633.

16. Céspedes L, Cornejo P. Prevalencia de Síntomas Premenstruales y Dismenorrea en Mujeres en Edad Fértil y su Relación con el Ausentismo Laboral. Tesis no publicada, Pontificia Universidad Católica de Chile, Santiago, Chile, 1997.

17. Burton W, Conti D, Chen Ch, Edington D, Schultz A. The Role of Health Risk Factors and Disease on
Worker Productivity. J Occup Environ Med 1999; 41(10): 863-77.

18. Koopman Ch, Pelletier K, Murray J, Sharda C, Berger M, Turpin R, Hackleman P, Gibson P, Holmes D, Bendel T. Stanford Presenteeism Scale: Health Status and Employee Productivity. J Occup Environ Med 2002; 44(1): 14-20.

19. Baladrón M, Toro V. Influencia del Síndrome Premenstrual en el Rendimiento Físico e Intelectual de Alumnas de Enfermería-Obstetricia y Medidas de Autocuidado que realizan. Tesis no publicada, Pontificia Universidad Católica de Chile, Santiago, Chile, 1999.

20. Silva M, Dias de Araújo e Silva C, Fleury G, Granzotto T, Dias T, Candido V. Síndrome PréMenstrual e Desempenho Escolar. Rev Bras Ginecol Obstet 2001; 23(7): 459-62.

21. Moya F. Mujer y Mercado Laboral. El difícil arte de trabajar. 2001. SERNAM. Hallado en: www.mujereschile.cl/conocedoras/ articulos.php?articulo=123\&area=trabajo. Acceso 10 de marzo 2005.

22. Marván M, Cortés-Iniestra S. Women's Beliefs About the Prevalence of Premenstrual Syndrome and Biases in Recall of Premenstrual Changes. Health Psychology 2001; 20(4): 276-80. 